\title{
SIMULATION MODEL OF INDUCTION HEATING IN COMSOL MULTIPHYSICS
}

\author{
Matúš OCILKA, Dobroslav KOVÁČ \\ Department of Theoretical and Industrial Electrical Engineering, Faculty of Electrical Engineering and Informatics, \\ Technical University of Košice, Letná 9, 04200 Košice, Slovak Republic, tel. +421 55602 2873, \\ e-mail: \{matus.ocilka, dobroslav.kovac\}@tuke.sk
}

\begin{abstract}
The induction heating phenomenon depends on various factors, making the problem highly nonlinear. The mathematical analysis of this problem in most cases is very difficult and it is reduced to simple cases. Other knowledge of induction heating systems is generated in production environments, but these trial-error procedures are long and expensive. The numerical models of induction heating problem are another approach to reduce abovementioned drawbacks. This paper deals with the simulation model of induction heating problem. The simulation model of induction heating system in COMSOL Multiphysics is created. The equivalent inductance and equivalent resistance of induction coil - workpiece coupling during heating process and temperature change for different parameters of AC current flowing through induction coil is obtained. In model, nonlinear electromagnetic and thermal properties of workpiece material are included. The simulation model provides essential information about processes ongoing in heated material. In order to verify the simulation model, the experimental measurements are made.
\end{abstract}

Keywords: COMSOL Multiphysics, induction heating, nonlinear material, numerical simulation

\section{INTRODUCTION}

The induction heating (IH) is unique, non - contact technique of heating electrically conductive materials. As its name implies, this technique relies on eddy currents that are internally induced in heating material. These eddy currents flow in electrically conductive material causing the primary mechanism of heat dissipation due to Joule's heat. This mechanism of heat generation is present in every electrically conductive material even non-magnetic one. The second mechanism of heat generation in workpiece is related with hysteresis losses and it is only present in magnetic materials [1].

The design of IH system includes knowing the processes ongoing in workpiece and induction coil. This includes the electromagnetic phenomena such as nonuniform distribution of magnetic flux density and current density in the workpiece and the coil, coupling between the workpiece and induction coil, the shape of the coil and workpiece. Heat generation, heat transfer in material and heat transfer between the material and external environment are other factors to be considered in induction heating phenomenon.

Abovementioned processes are all related to physical properties of material to be heated. These properties include the electromagnetic and thermal properties of material and they are highly depended on its temperature. The amount of heat generated in workpiece depends on the magnitude of electric current flowing through the coil and heating depth depends on its frequency.

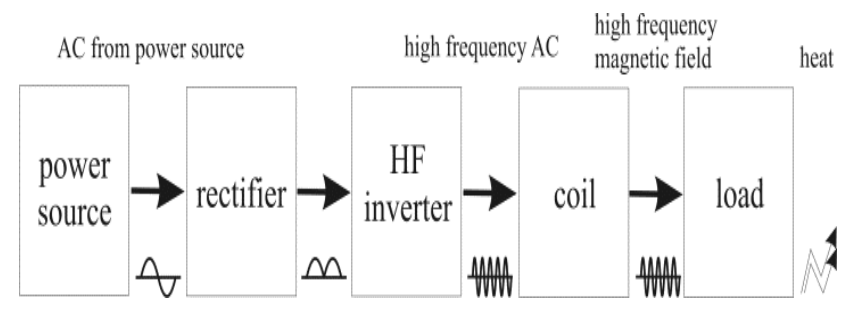

Fig. 1 IH system
Considering the previous facts, the $\mathrm{IH}$ phenomenon is a complex problem, thus numerical simulations seem to be a useful tool for the design and the investigation of IH systems [4].

The basic IH system consists of source of AC current, induction coil a workpiece itself (Fig.1). In order to design the source of AC current, the equivalent resistance $\left(R_{e q}\right)$ and equivalent inductance $\left(L_{e q}\right)$ of induction coil - workpiece coupling needs to be estimated based on the series model of this coupling (Fig. 2) [5].

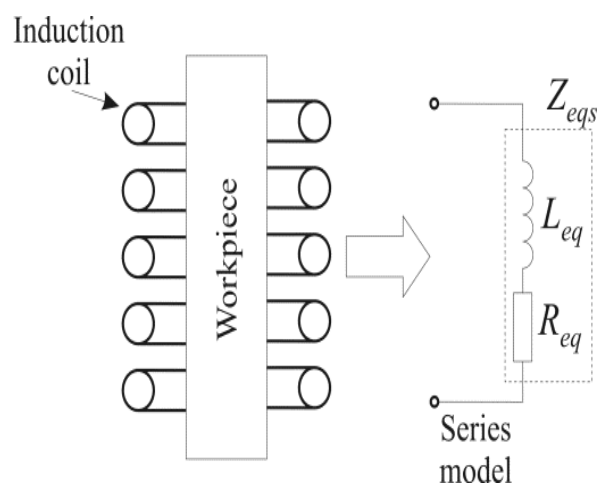

Fig. 2 Series model of induction coil - workpiece coupling

In this paper, the model of induction heating using finite elements method of solution is introduced which considers non-linear properties of heated material. This model allows observing the relations that are present in workpiece and induction coil during the heating process.

The laboratory model of converter is developed and experimental measurements of temperature are compared with simulation results.

\section{MATHEMATICAL MODEL OF IH}

The induction heating phenomenon consists of coupled problem combining the electromagnetic problem and thermal problem including the radiation and convective transition considering the material properties [4]. 


\subsection{Electromagnetic problem}

The problem of electromagnetic analysis on a macroscopic level is that of solving Maxwell's equations and constitutive relationships subject to certain boundary conditions. For general time - varying fields, Maxwell's equation can be written as [3], [8]:

$$
\begin{aligned}
& \nabla \times \mathbf{H}=\mathbf{J}+\frac{\partial \mathbf{D}}{\partial t} \\
& \nabla \times \mathbf{E}=-\frac{\partial \mathbf{B}}{\partial t} \\
& \nabla . \mathbf{D}=\rho \\
& \nabla . \mathbf{B}=0
\end{aligned}
$$

where: $\mathbf{H}$ - magnetic field intensity, $\mathbf{J}$ - current density, B - magnetic flux density, $\mathbf{E}$ - electric field intensity,

D - electric flux density, $\rho$ - electric charge density.

To obtain a closed system, the equations include constitutive relationships that describe the macroscopic properties of the medium:

$$
\begin{aligned}
& \mathbf{D}=\varepsilon_{0} \varepsilon_{r} \mathbf{E} \\
& \mathbf{B}=\mu_{0} \mu_{r} \mathbf{H} \\
& \mathbf{J}=\sigma \mathbf{E}
\end{aligned}
$$

where: $\varepsilon_{0}$ - vacuum permittivity, $\varepsilon_{r}$ - relative permittivity, $\mu_{0}$ - vacuum permeability, $\mu_{r}-$ relative permeability, $\sigma$ - electrical conductivity.

The simulation model of induction heating created in $2 \mathrm{D}$ axisymmetric domain is shown in Fig. 3. The solution domain $\Omega$ is divided into three subdomains: air $\left(\Omega_{3}\right)$, coil $\left(\Omega_{2}\right)$ and workpiece domain $\left(\Omega_{1}\right)$. For the workpiece a bolt M12x60 mm made of carbon steel was chosen.

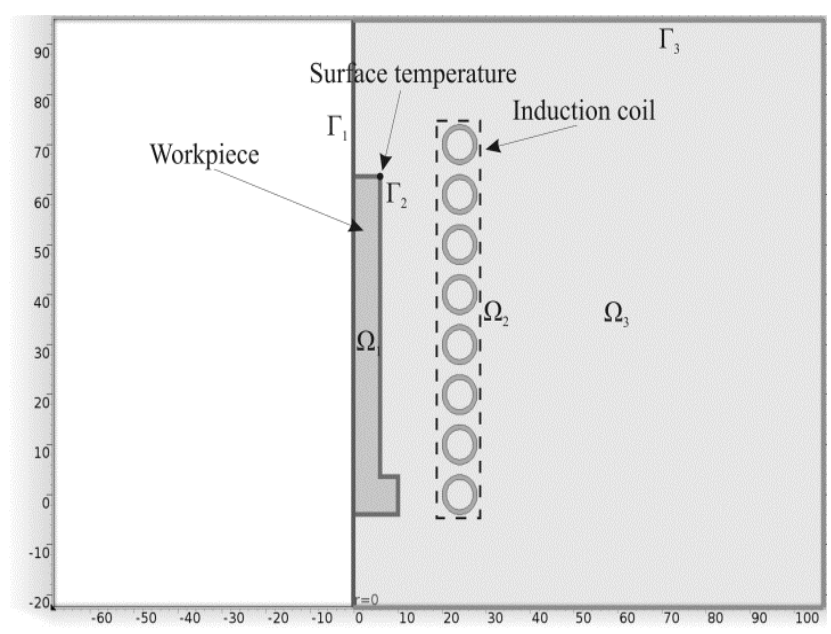

Fig. 3 IH model in COMSOL Multiphysics

The material of subdomain $\Omega_{1}$ is medium carbon steel. Both domains $\Omega_{1}$ and $\Omega_{2}$ are surrounded with nonconductive air region $\Omega_{3}$. Equations for this particular problem for frequency - transient analysis can be written as follows [3].
Subdomain $\Omega_{1}, \Omega_{3}$ :

$$
\begin{aligned}
& \left(j \omega-\omega^{2} \varepsilon_{0} \varepsilon_{r}\right) \mathbf{A}+\nabla \times\left(\mu_{0}^{-1} \mu_{\mathbf{r}}^{-1} \mathbf{B}\right)=\mathbf{J}_{\mathbf{e}} \\
& \mathbf{B}=\nabla \times \mathbf{A}
\end{aligned}
$$

where: $j$ - imaginary unit, $\omega-$ angular frequency, $\mathbf{A}$ - magnetic vector potential, $\mathbf{J}_{\mathbf{e}}-$ current density.

\section{Subdomain $\Omega_{2}$ :}

$\left(j \omega-\omega^{2} \varepsilon_{0} \varepsilon_{r}\right) \mathbf{A}+\nabla \times \mathbf{H}=\mathbf{J}_{\mathbf{e}}$

$\int_{\Omega} \mathbf{J}_{i} \mathbf{\Phi} d S=I_{\text {coil }}$

$\mathbf{J}_{\mathbf{e}}=\frac{\sigma V_{i}}{2 \pi r} \boldsymbol{\Phi}$

$V_{\text {coil }}=\sum_{i} V_{i}$

where: $\Phi$ - magnetic flux, $I_{\text {coil }}$ - coil current, $V_{i}$ - voltage of one turn of the coil, $V_{\text {coil }}$ - voltage of the coil.

\section{Boundary $\Gamma_{3}$ :}

$\mathbf{n} \times \mathbf{A}=0$

where: $\mathbf{n}$ - outward normal from medium.

\subsection{Thermal problem}

The fundamental law describing all heat transfer is the first law of thermodynamics. It is commonly referred as the principle of conservation energy. In induction heating, all three modes of heat transfer - conduction, convection and radiation are present [3].

The law that describes heat transfer by conduction is known as Fourier's law [1]:

$$
q_{\text {cond }}=-k \nabla T
$$

where: $q_{\text {cond }}$ - heat flux by conduction, $k$ - thermal conductivity, $T$ - temperature.

In this mode of heat transfer, heat is transferred from high - temperature regions towards low - temperature region.

On the other hand heat transfer by convection carries out only in gas or fluid and it is described by the Newton's law as follows [1]:

$q_{\text {conv }}=-h\left(T_{\text {amd }}-T\right)$

where: $q_{c o n v}-$ heat flux by convection, $h$ - convection heat transfer coefficient, $T_{a m b}-$ ambient temperature.

This law describes the heat transfer between the surface of the heated worpiece and ambient area. The heat transfer rate is proportional to the temperature difference and heat transfer coefficient.

Last mode of heat transfer is known as heat radiation. The heat is transferred from the hot surface of the 
workpiece to the ambient area including nonmaterial region (vacuum). Heat transfer by radiation takes place through the transport of photons. Participating media absorb, emit and scatter photons. Opaque surface absorb or reflect them [3]. The basic law that governs this mode of heat transfer is known as Stefan - Boltzmann's law [1]:

$q_{\text {rad }}=8 \sigma\left(T_{s}^{4}-T_{a m b}{ }^{4}\right)$

where: $q_{\text {rad }}$ - radiation loss density, $\varepsilon$ - surface emissivity, $\sigma-$ Stefan - Boltzmann constant, $T_{s}$ - surface temperature.

According to previous modes of heat transfer, the equations for subdomains and boundaries can be written as follows [3]:

\section{Subdomain $\Omega_{1}$ :}

$\rho C_{p} \frac{\partial T}{\partial t}+\nabla \cdot(-k \nabla T)=Q$

$Q=Q_{r h}+Q_{m l}$

where: $C_{p}$ - heat capacity, $\rho$ - density, $Q-$ heat sources other than viscous heating, $Q_{r h}$ - resistive losses, $Q_{m l}-$ magnetic losses.

\section{Boundary $\Gamma_{3}$ :}

$-\mathbf{n} .(-k \nabla T)=h\left(T_{e x t}-T\right)$

$-\mathbf{n} .(-k \nabla T)=\varepsilon \sigma\left(T_{a m b}{ }^{4}-T^{4}\right)$

\subsection{Electromagnetic and thermal properties of workpiece material}

Electromagnetic and heat transfer phenomenon are highly interrelated because the physical properties of heated metals. These properties of metals are nonlinear functions of magnetic field intensity, temperature, chemical composition and other factors [1].

This mathematical model considers the main properties that have the most significant impact on induction heating technology. These properties are: relative permeability $\mu_{r}$, electric resistivity $\rho$, thermal conductivity $k$ and heat capacity $C_{p}$. Values of these properties used in simulation for carbon steel depending on temperature are listed in Tab.1. The Curie temperature was set to $T_{c}=740{ }^{\circ} \mathrm{C}$.

It should be noted that surface emissivity $\varepsilon$ and convection heat transfer coefficient $h$ are temperature depended as well (other factors that influence $\varepsilon$ and $h$ are: viscosity, surface condition, etc...), but in this model these properties as well as conductivity of copper of induction coil (subdomain $\Omega_{2}$ ) remain constant during heating process.

The value of emissivity was set to $\varepsilon=0,75$, convection heat transfer coefficient is $h=15 \mathrm{~W} \cdot \mathrm{m}^{-2} \cdot \mathrm{K}^{-1}$ and value of electric conductivity of the coil is $\sigma=5,99.10^{7}$ S.m $\mathrm{m}^{-1}$.
Table 1 Electromagnetic and thermal properties of medium carbon steel depending on temperature

$\begin{array}{ccccc}T & k & C_{p} & \rho & \mu_{r} \\ {[\mathrm{~K}]} & {[\mathrm{W} /(\mathrm{m} . \mathrm{K})]} & {[\mathrm{J} /(\mathrm{kg} . \mathrm{K})]} & {[\Omega \cdot \mathrm{m}]} & {[-]} \\ 293 & 52 & 450 & 1,70.10^{-7} & 218 \\ 373 & 51 & 490 & 2,32.10^{-7} & - \\ 473 & - & - & 3,10.10^{-7} & - \\ 573 & 46 & 570 & 4,10.10^{-7} & 202 \\ 673 & - & - & 5,05.10^{-7} & - \\ 773 & 38 & 680 & - & - \\ 873 & - & - & 7,72.10^{-7} & 164 \\ 973 & 30 & - & 9,35.10^{-7} & 81 \\ 1013 & - & - & 1,03.10^{-6} & 27 \\ 1073 & - & - & 1,13.10^{-6} & 1 \\ 1273 & 27 & 570 & - & 1 \\ 1473 & 27 & 570 & 1,23.10^{-6} & 1\end{array}$

\section{SIMULATION RESULTS}

The simulation results of induction heating model for coil current $I_{\max }=50 \mathrm{~A}$ and frequency $f=100 \mathrm{kHz}$ are shown in Fig. 4. The simulation time was set to $10 \mathrm{~min}$. The maximum temperature reached is $611^{\circ} \mathrm{C}$.

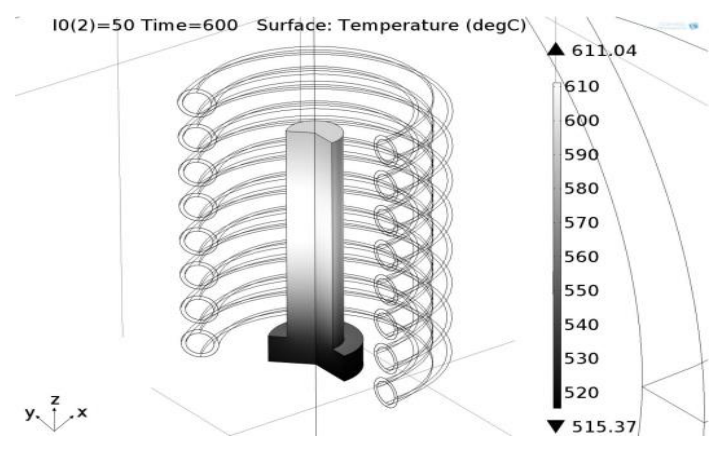

Fig. 4 Temperature of workpiece after $600 \mathrm{~s}$ of heating

The surface temperature of workpiece (shown in Fig. 3) is depicted in Fig. 5.

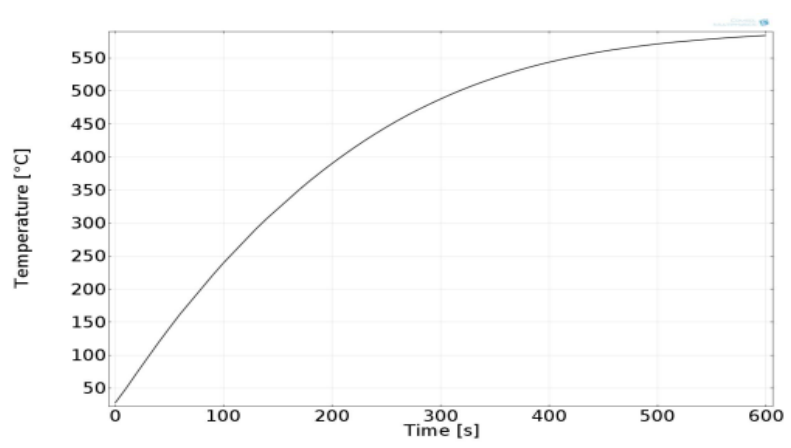

Fig. 5 Surface temperature of workpiece during heating process for $I_{\max }=50 \mathrm{~A}$ and $f=100 \mathrm{kHz}$ 
The $R_{e q}$ and $L_{e q}$ of coil - worpiece model is shown in Fig. 6 and Fig. 7, respectively.

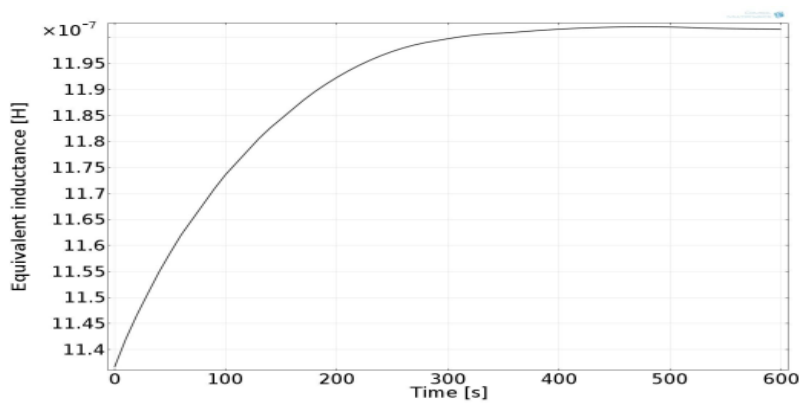

Fig. 6 Equivalent inductance during heating process for $I_{\max }=50 \mathrm{~A}$ and $f=100 \mathrm{kHz}$

The initial value of $L_{e q}$ is $1.14 \mu \mathrm{H}$. After $600 \mathrm{~s}$ the value settled down to $L_{e q}=1.20 \mu \mathrm{H}$.

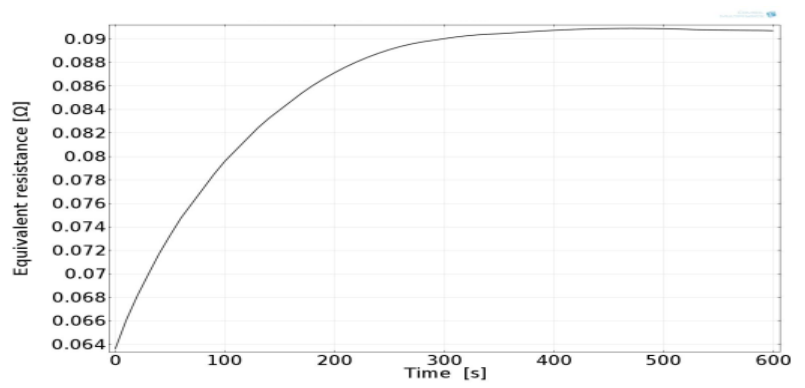

Fig. 7 Equivalent resistance during heating process for $I_{\max }=50 \mathrm{~A}$ and $f=100 \mathrm{kHz}$

The value of $R_{e q}$ has similar graph as for $L_{e q}$. In this case the initial value is $R_{e q}=0.064 \Omega$ and maximum value reached is $R_{e q}=0.091 \Omega$.

\section{EXPERIMENTAL RESULTS}

Considering the previous results the real induction coil and source of AC current were built. Using the simulation results of $R_{e q}$ and $L_{e q}$ the frequency of resonant circuit was calculated, since the converter used in experimental measurements utilizes an LCL resonant circuit [6], [7]. During the heating of workpiece $L_{e q}$ and $R_{e q}$ varies, thus the resonant frequency range needs to be calculated.

Calculated resonant frequency of this circuit varies within range $f_{r}=103.3-105.2 \mathrm{kHz}$.

The experimental measurement of temperature for above-mentioned frequency range and coil current of $I_{\max }=50 \mathrm{~A}$ is shown in Fig. 8.

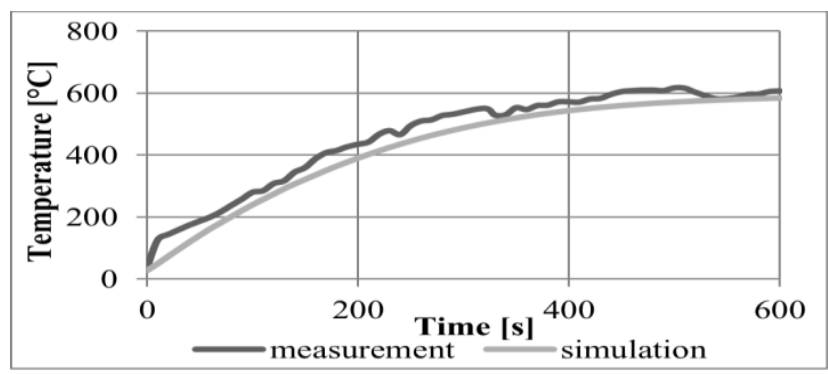

Fig. 8 Comparison between simulation and experimental measurement for $I_{\max }=50 \mathrm{~A}$
The measuring point is again depicted in Fig. 3. The difference between the simulation and experimental measurement is shown in Fig. 9.

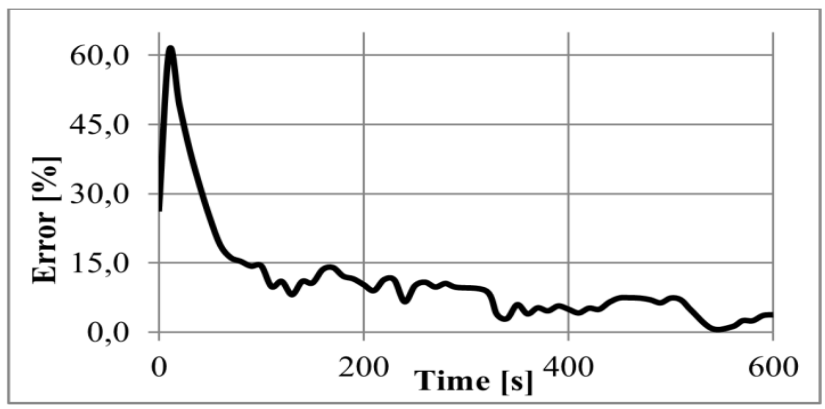

Fig. 9 Relative error between simulation and experimental measurement

The relative error in dynamic states reached value of 61 $\%$. This could be caused by constant value of surface emissivity and convection heat transfer coefficient. Another reason is that frequency of measurement $\mathrm{AC}$ current varied within range $103.3-105.2 \mathrm{kHz}$ and frequency used in simulation was set to $100 \mathrm{kHz}$. On the other hand, in steady - state, error is approximately $5 \%$.

In the Fig. 10 the induction coil used in experimental measurements is shown.

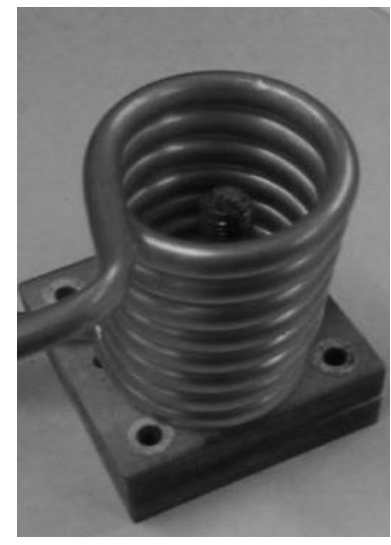

Fig. 10 Real induction coil

\section{CONCLUSIONS}

In this paper the simulation model of induction heating considering the non-linear properties of metal is developed. The simulation model was compared with experimental results. The experimental measurements show that chosen approach is acceptable for designing systems that utilize induction heating technology.

\section{ACKNOWLEDGMENTS}

The paper has been prepared under support of Slovak grant project KEGA No. 015TUKE-4/2015.

\section{REFERENCES}

[1] RUDNEV, V. - LOVELESS, D. - COOK, R. BLACK, M.: Handbook of Induction Heating, Marcel Dekker, Inc., New York, USA, 2003, ISBN 0-82470848-2 
[2] ZIN, S. - SEMIATIN, S. L.: Elements of Induction Heating: Design, Control, and Applications, ASM International, Materials Park, Ohio, USA, 1988

[3] COMSOL Multiphysics User's Guide, Version 4.3a, Comsol AB, October, 2012

[4] LIU, H. - RAO, J.: Coupled Modelling of Electromagnetic-Thermal Problem in Induction Heating Process Considering Material Properties, Information Engineering and Computer Science, 2009. ICIECS 2009. International Conference on, vol., no., pp.1, 4, 19-20 Dec. 2009

[5] SEGURA, G. M.: Induction Heating Converter's Design, Control and Modelling Applied to Continuous Wire Heating, doctoral thesis, Barcelona, 2012

[6] DIECKERHOFF, S. - RUAN, M. J. - DE DONCKER, R. W.: Design of an IGBT-based LCLresonant inverter for high-frequency induction heating, Industry Applications Conference, 1999. Thirty-Fourth IAS Annual Meeting, Conference Record of the 1999 IEEE, vol.3, no., pp.2039,2045 vol.3, 1999

[7] CHUDJUARJEEN, S. - SANGSWANG, A. KOOMPAI, C.: An Improved Resonant Inverter for Induction-Heating Applications With Asymmetrical Control, Industrial Electronics, IEEE Transactions on , vol.58, no.7, pp.2915,2925, July 2011

[8] MAYER, D.: Aplikovaný elektromagnetizmus, druhé vydanie, KOPP, České Budějovice, 2012,
ISBN: 978-80-7232-436-1

Received March 10, 2015 accepted March 26, 2015

\section{BIOGRAPHIES}

Matúš Ocilka was born on 17.4.1986. In 2010 he graduated (MSc) at the Department of Electrical Engineering and Mechatronics of the Faculty of Electrical Engineering and Informatics at Technical University of Košice. Currently he is a PhD student at the Department of Theoretical and Industrial Electrical Engineering; the title of his thesis is "High frequency converter for induction heating". The field of interest is mainly focused in power electronics, numeric simulations, and process automation.

Dobroslav Kováč - He finished his studies in 1985 at the Technical University of Košice, Department of Electrical Drives, area - Power electronics with excellent evaluation. Then he worked as a research worker at the Department of Electrical Drives. His research work was focused on the practical application of new power semiconductor devices. In 1989 he got the Award of the Minister of Education for the Development of Science and Technology. From 1991 he has worked as assistant lecturer at the Department of Theoretical Electrical Engineering and Electrical Measurement. He got his doctoral diploma in 1992 for the work on the field of power electronics. From 2000 he has worked as professor and his working interest is now focused mainly on the field of computer simulation, industrial systems, smart power electronics and automated computer measuring. 